\title{
COMMON SOURCE OF NUMEROUS THETA FUNCTION IDENTITIES
}

\author{
CHU WENCHANG \\ Department of Applied Mathematics, Dalian University of Technology, Dalian 116024, P. R. China
}

(Received 27 January, 2006; accepted 27 September, 2006)

\begin{abstract}
Motivated by the recent work due to Warnaar (2005), two new and elementary proofs are presented for a very useful $q$-difference equation on eight shifted factorials of infinite order. As the common source of theta function identities, this $q$ difference equation is systematically explored to review old and establish new identities on Ramanujan's partition functions. Most of the identities obtained can be interpreted in terms of theorems on classical partitions.
\end{abstract}

2000 Mathematics Subject Classification. Primary 05A30. Secondary 14K25.

For two indeterminates $q$ and $z$ with $|q|<1$, the $q$-shifted factorial of infinite order and the theta function are defined respectively by

$$
(z ; q)_{\infty}=\prod_{n=0}^{\infty}\left(1-z q^{n}\right) \quad \text { and } \quad\langle z ; q\rangle_{\infty}=(z ; q)_{\infty}(q / z ; q)_{\infty}
$$

Their product forms are abbreviated respectively as

$$
\begin{aligned}
& {[\alpha, \beta, \cdots, \gamma ; q]_{\infty}=(\alpha ; q)_{\infty}(\beta ; q)_{\infty} \cdots(\gamma ; q)_{\infty}} \\
& \langle\alpha, \beta, \cdots, \gamma ; q\rangle_{\infty}=\langle\alpha ; q\rangle_{\infty}\langle\beta ; q\rangle_{\infty} \cdots\langle\gamma ; q\rangle_{\infty}
\end{aligned}
$$

Recently, a very useful $q$-difference equation on eight shifted factorials of infinite order was established in [11, Theorem 1.1], where its applications to Ramanujan's congruences on the partition function are investigated. The purpose of this paper is twofold. First, we shall present two new and elementary proofs for that $q$-difference equation, inspired by Warnaar's recent paper [23]. Second, we shall explore its applications along another direction: theta function identities. We shall systematically review old and establish new theta function identities from that $q$-difference equation, which turns out to be a natural source for theta function identities.

The contents of the paper will be as follows. The first section will enunciate the $q$-difference equation on shifted factorials and its main implications. The two new and elementary proofs will be presented in the second section. The third section will be dedicated to its applications to various theta function identities.

Current Address: Dipartimento di Matematica, Università degli Studi di Lecce, Lecce-Arnesano, P. O. Box 193, 73100 Lecce, ITALIA. e-mail: chu.wenchang@unile.it

Work carried out during the visit to Dalian University of Technology (2005). 
1. The Main Theorem and Implications. For the difference of eight shifted factorials of infinite order, we have the following fundamental result.

THEOREM 1 (Theta function identity [10] and [11]). For five complex parameters $a, b, c, d$, e satisfying $a^{2}=b c d e$, there holds the theta function identity

$$
\langle a / b, a / c, a / d, a / e ; q\rangle_{\infty}-\langle b, c, d, e ; q\rangle_{\infty}=b\langle a, a / b c, a / b d, a / b e ; q\rangle_{\infty}
$$

This identity appeared explicitly for the first time in [10]. The proof given there by Chapman is equivalent to the following theta function identity

$$
\begin{aligned}
& \vartheta(a-b) \vartheta(a-c) \vartheta(a-d) \vartheta(a-e)-\vartheta(b) \vartheta(c) \vartheta(d) \vartheta(e) \\
& =\vartheta(a) \vartheta(a-b-c) \vartheta(a-b-d) \vartheta(a-b-e)
\end{aligned}
$$

where the theta function is defined (cf. [25, $§ 21.11])$ by

$$
\vartheta(z)=2 q^{\frac{1}{8}} \sum_{n=0}^{\infty}(-1)^{n} q^{\left(\begin{array}{c}
n+1 \\
2
\end{array}\right)} \sin (2 n+1) z .
$$

Liu [18, Thm 4] has independently rediscovered the theta function identity just displayed by applying the Cauchy residue theorem to theta functions within a parallelogram. In particular, the following three parameter version of Theorem 1 has been found by Warnaar [23, Eq. 3]

$$
\langle-c,-a c,-b c,-a b c ; q\rangle_{\infty}-\langle c, a c, b c, a b c ; q\rangle_{\infty}=c\left\langle-q,-a,-b,-a b c^{2} ; q\right\rangle_{\infty}
$$

which generalizes the theta function identity due to Farkas and Kra [15] (see also Warnaar [23, Eq. 1]):

$$
\left(-q ; q^{2}\right)_{\infty}\left(-q^{7} ; q^{14}\right)_{\infty}-\left(q ; q^{2}\right)_{\infty}\left(q^{7} ; q^{14}\right)_{\infty}=2 q\left(-q^{2} ; q^{2}\right)_{\infty}\left(-q^{14} ; q^{14}\right)_{\infty}
$$

Even Warnaar found only a particular case of Theorem 1; his proofs are inspiring, and stimulated the author to present two new and elementary proofs of Theorem 1.

Theorem 1 was originally demonstrated by telescoping the particular case $a^{2}=$ $b c d e$ of Bailey's very well-poised bilateral summation formula (cf. [16, §5.3])

$$
\begin{aligned}
& { }_{6} \psi_{6}\left[\begin{array}{c}
q a^{1 / 2},-q a^{1 / 2}, \quad b, \quad c, \quad d, \quad e|c| q a^{2} \\
a^{1 / 2},-a^{1 / 2}, q a / b, q a / c, q a / d, q a / e
\end{array}\right] \\
& =\frac{[q, q a, q / a, q a / b c, q a / b d, q a / b e, q a / c d, q a / c e, q a / d e ; q]_{\infty}}{\left[q a / b, q a / c, q a / d, q a / e, q / b, q / c, q / d, q / e, q a^{2} / b c d e ; q\right]_{\infty}}
\end{aligned}
$$

which has been reproduced in [11], where its applications to the symmetric differences on theta functions due to Ewell $[\mathbf{1 3}, \mathbf{1 4}]$ and Berndt et al. [5], as well as Ramanujan's congruences on the partition function, are systematically investigated. 
In order to facilitate the subsequent applications, we highlight the following reduced forms of Theorem 1. First, letting

$$
\begin{aligned}
a & =u v w, \\
b & =-1, \\
c & =-u v, \\
d & =-u w, \\
e & =-v w,
\end{aligned}
$$

we may reformulate (1.1) as an additive theorem on theta functions.

THEOREM 2. For three variables $u, v$ and $w$, there holds the theta function identity

$$
\langle-u,-v,-w,-u v w ; q\rangle_{\infty}+\langle u, v, w, u v w ; q\rangle_{\infty}=2(-q ; q)_{\infty}^{2}\langle-u v,-u w,-v w ; q\rangle_{\infty} .
$$

Second, we show the following pair of interesting and useful identities.

Proposition 3. For two variables $x$ and $y$, there hold two theta function identities:

$$
\langle-x,-y ; q\rangle_{\infty} \pm\langle x, y ; q\rangle_{\infty}= \begin{cases}2(-q ; q)_{\infty}^{2}\left\langle-x y,-q x / y ; q^{2}\right\rangle_{\infty}, & \text { “+”; } \\ 2 x(-q ; q)_{\infty}^{2}\left\langle-q x y,-q^{2} x / y ; q^{2}\right\rangle_{\infty}, & \text { “-”. }\end{cases}
$$

Proof. The identity corresponding to "-" follows from Theorem 1 with the parameter setting $a \rightarrow-x y, b \rightarrow x, c \rightarrow y$ and $d, e \rightarrow \pm \sqrt{-x y}$. Similarly, the identity corresponding to " + " is obtained from Theorem 2 with the parameters being specified by $u, v= \pm \sqrt{-y / x}$ and $w=x$.

Finally, specifying parameters in Theorem 1 with

$$
\left.\begin{array}{l}
b \rightarrow u / v \\
c \rightarrow-u v \\
d \rightarrow q^{1 / 2} \\
e \rightarrow-q^{1 / 2}
\end{array}\right\} \quad a=-q^{1 / 2} u
$$

we obtain the $q$-difference equation:

$$
\begin{aligned}
\frac{u}{v}\left\langle q^{1 / 2} / u,-q^{1 / 2} u, v,-v ; q\right\rangle_{\infty} & =\left\langle u,-u, q^{1 / 2} / v,-q^{1 / 2} v ; q\right\rangle_{\infty} \\
& -\left\langle u / v,-u v, q^{1 / 2},-q^{1 / 2} ; q\right\rangle_{\infty}
\end{aligned}
$$

which is equivalent to the following identity due to Ewell.

Corollary 4 (Ewell [13, Theorem 1.1] and [14, Eq. 2.1]).

$$
v\left\langle u^{2}, q v^{2} ; q^{2}\right\rangle_{\infty}-u\left\langle v^{2}, q u^{2} ; q^{2}\right\rangle_{\infty}=\frac{v}{(-q ; q)_{\infty}^{2}}\langle u / v,-u v ; q\rangle_{\infty}
$$

Ewell $[12,13,14]$ explored differences of triple products. More applications of this formula to theta function identities may be found in the recent paper [11, §3].

2. New Proofs of Theorem 1. Inspired by Warnaar [23], we give two new and elementary proofs of Theorem 1. 
2.1. The first new proof of Theorem 1. Performing the parameter replacements $c \rightarrow z$ and $e \rightarrow a^{2} / b d z$ in the equation (1.1), we may restate the result as the equation $F(z):=\mathcal{L}(z) / \mathcal{R}(z)=1$, where

$$
\begin{aligned}
\mathcal{L}(z) & :=\langle a / b, a / d, a / z, b d z / a ; q\rangle_{\infty}-\left\langle b, d, z, a^{2} / b d z ; q\right\rangle_{\infty} \\
\mathcal{R}(z) & :=b\langle a, a / b d, a / b z, d z / a ; q\rangle_{\infty} .
\end{aligned}
$$

It is not hard to check that

$$
\mathcal{L}(q z)=\mathcal{L}(z) \frac{a^{2}}{q b d z^{2}} \quad \text { and } \quad \mathcal{R}(q z)=\mathcal{R}(z) \frac{a^{2}}{q b d z^{2}} .
$$

We have therefore

$$
F(z)=F(q z)=F\left(q^{2} z\right)=\cdots .
$$

Possible poles of $F(z)$ are given by zeros of $\mathcal{R}(z)$, which consist of $z=q^{n} a / b$ and $z=q^{n} a / d$ with $n \in \mathbb{Z}$. However $\mathcal{L}\left(q^{n} a / b\right)=0$ for $n \in \mathbb{Z}$, which is justified as follows:

$$
\begin{aligned}
\mathcal{L}\left(q^{n} a / b\right) & =\left\langle a / b, a / d, q^{-n} b, q^{n} d ; q\right\rangle_{\infty}-\left\langle b, d, q^{n} a / b, q^{-n} a / d ; q\right\rangle_{\infty} \\
& =\langle b, d, a / b, a / d ; q\rangle_{\infty}\left\{\left[\begin{array}{c}
q^{-n} b, q^{1-n} / d \\
q / b, d
\end{array} \mid q\right]_{n}-\left[\begin{array}{c}
q^{1-n} b / a, q^{-n} a / d \\
a / b, q d / a
\end{array} \mid q\right]_{n}\right\} \\
& \left.=\langle b, d, a / b, a / d ; q\rangle_{\infty}\left\{\left[\begin{array}{c}
q / b, d \\
q / b, d
\end{array} \mid q\right]_{n}-\left[\begin{array}{c}
a / b, q d / a \\
a / b, q d / a
\end{array}\right]\right]_{n}\right\}\left(q^{-n} \frac{b}{d}\right)^{n}=0 .
\end{aligned}
$$

Observing that $\mathcal{L}(z)$ is symmetric with respect to $b$ and $d$, we have also $\mathcal{L}\left(q^{n} a / d\right)=0$ for $n \in \mathbb{Z}$. Therefore $F(z)$ is an entire and bounded function and must be constant thanks to Liouville's theorem. The constant is unity, since

$$
\begin{aligned}
F(z) & \equiv F(1)=\frac{\mathcal{L}(1)}{\mathcal{R}(1)}=\frac{\langle a, a / b, a / d, b d / a ; q\rangle_{\infty}}{b\langle a, a / b, d / a, a / b d ; q\rangle_{\infty}} \\
& =\frac{(1-a / d)(1-b d / a)}{b(1-d / a)(1-a / b d)} \frac{\langle a, a / b, a / d, b d / a ; q\rangle_{\infty}}{\langle a, a / b, a / d, b d / a ; q\rangle_{\infty}}=1 .
\end{aligned}
$$

$\S$ 2.2. The second new proof of Theorem 1. Recall the Jacobi triple product identity (cf. $[16, \S 1.6]$ for example),

$$
\sum_{n=-\infty}^{+\infty}(-1)^{n} q^{\left(\begin{array}{c}
n \\
2
\end{array}\right)} x^{n}=[q, x, q / x ; q]_{\infty} .
$$

Replacing $e$ by $a^{2} / b c d$ and then multiplying both sides by $(q ; q)_{\infty}^{4}$, we can expand (1.1) in formal Laurent series as follows:

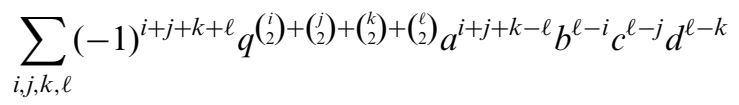

$$
\begin{aligned}
& -\sum_{i, j, k, \ell}(-1)^{i+j+k+\ell} q^{\left(\begin{array}{l}
i \\
2
\end{array}\right)+\left(\begin{array}{l}
j \\
2
\end{array}\right)+\left(\begin{array}{l}
k \\
2
\end{array}\right)+\left(\begin{array}{l}
\ell \\
2
\end{array}\right) a^{2 \ell} b^{i-\ell} c^{j-\ell} d^{k-\ell}} \\
& =[q, a, q / a ; q]_{\infty} \sum_{i, j, k}(-1)^{i+j+k} q^{\left(\begin{array}{c}
i \\
2
\end{array}\right)+\left(\begin{array}{l}
j \\
2
\end{array}\right)+\left(\begin{array}{l}
k \\
2
\end{array}\right) a^{i+j-k} b^{1-i-j} c^{k-i} d^{k-j} .}
\end{aligned}
$$


Extracting the coefficients of $b^{B} c^{C} d^{D}$ from both sides of this equation and then

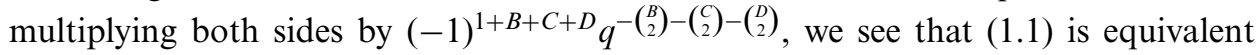
to the identity

$$
\begin{aligned}
& \sum_{\ell}\left\{q^{4\left(\begin{array}{l}
\ell \\
2
\end{array}\right)+\ell(B+C+D)} a^{2 \ell}-q^{4\left(\begin{array}{l}
\ell \\
2
\end{array}\right)+(1-\ell)(B+C+D)} a^{2 \ell-B-C-D}\right\} \\
& = \begin{cases}0, & B+C+D \text { - even; } \\
(-a)^{\frac{1-B-C-D}{2}}[q, a, q / a ; q]_{\infty} q^{-\left({ }^{(B+C+D-1) / 2}\right),} & B+C+D \text { - odd } .\end{cases}
\end{aligned}
$$

Depending on the parity, let $B+C+D=2 m+\delta$ with $\delta=0,1$. Shifting the summation index by $\ell \rightarrow \ell+m$ for the second sum and keeping the first invariant, we can reduce the sum displayed in (2.3a) as follows:

$$
\begin{aligned}
& \mathrm{Eq}(2.3 \mathrm{a})=\sum_{\ell}\left\{q^{4\left(\begin{array}{l}
\ell \\
2
\end{array}\right)+\ell(2 m+\delta)} a^{2 \ell}-q^{4\left(\begin{array}{l}
\ell \\
2
\end{array}\right)+(1-\ell)(2 m+\delta)} a^{2 \ell-2 m-\delta}\right\} \\
& =\sum_{\ell}\left\{q^{4\left(\begin{array}{c}
\ell \\
2
\end{array}\right)+\ell(2 m+\delta)} a^{2 \ell}-q^{4\left(\begin{array}{c}
\ell+m \\
2
\end{array}\right)+(1-\ell-m)(2 m+\delta)} a^{2 \ell-\delta}\right\}
\end{aligned}
$$

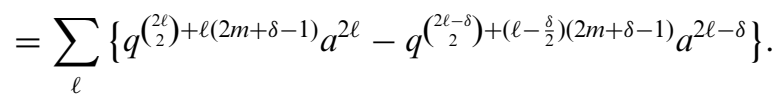

For $\delta=0$, the last sum vanishes, which coincides with (2.3b). When $\delta=1$, we can evaluate it by means of Jacobi's triple product identity as follows:

$$
\begin{aligned}
& \mathrm{Eq}(2.3 \mathrm{a})=\sum_{\ell}\left\{q^{\left(\begin{array}{c}
2 \ell \\
2
\end{array}\right)+2 \ell m} a^{2 \ell}-q^{\left(\begin{array}{c}
2 \ell-1 \\
2
\end{array}\right)+(2 \ell-1) m} a^{2 \ell-1}\right\} \\
& =\sum_{\ell}(-1)^{\ell} q^{\left(\frac{\ell}{2}\right)+\ell m} a^{\ell}=\left[q, q^{m} a, q^{1-m} / a ; q\right]_{\infty} \\
& =(-1)^{m} q^{-\left(\begin{array}{c}
m \\
2
\end{array}\right)} a^{-m}[q, a, q / a ; q]_{\infty} .
\end{aligned}
$$

This is consistent again with (2.3b) for the case $B+C+D=2 m+1$.

3. Theta Function Identities. Theorem 1 may be considered as a natural source of theta function identities, which will be exemplified in this section. For the presence of four free parameters, we shall specialize Theorem 1 to numerous theta function identities on Ramanujan's functions. On account of the generating functions for partitions (cf. Andrews [1, Chapter 2]), most of the theta function identities dealt with in this section can be interpreted in terms of classical theorems on partition enumerations. This aspect will not be covered in this paper. 
In order to facilitate the subsequent reference, we reproduce Ramanujan's notation on theta functions (cf. Berndt [3, Chapter 16], [4, Chapter 2] and Watson [24]):

$$
\begin{aligned}
f(a, b) & :=[a b,-a,-b ; a b]_{\infty} \quad \text { with } \quad f(-q):=f\left(-q,-q^{2}\right)=(q ; q)_{\infty} \\
\phi(q) & :=f(q, q)=\left[q^{2},-q,-q ; q^{2}\right]_{\infty} \quad \text { with } \quad \phi(-q)=\frac{(q ; q)_{\infty}}{(-q ; q)_{\infty}} \\
\psi(q) & :=f\left(q, q^{3}\right)=\frac{\left(q^{2} ; q^{2}\right)_{\infty}}{\left(q ; q^{2}\right)_{\infty}} \quad \text { and } \quad \chi(q):=\left(-q ; q^{2}\right)_{\infty} \\
G(q) & :=\frac{1}{\left\langle q ; q^{5}\right\rangle_{\infty}}=\sum_{n=0}^{+\infty} \frac{q^{n^{2}}}{(q ; q)_{n}} \quad \text { and } \quad H(q):=\frac{1}{\left\langle q^{2} ; q^{5}\right\rangle_{\infty}}=\sum_{n=0}^{+\infty} \frac{q^{n^{2}+n}}{(q ; q)_{n}} .
\end{aligned}
$$

$\S 3.1$. Seven easier identities. We review some familiar identities due to Jacobi.

EXAMPLE 1 Berndt [3, Entry 25, P 40].

$$
\begin{aligned}
& \left(-q ; q^{2}\right)_{\infty}+\left(q ; q^{2}\right)_{\infty}=\frac{2}{\left(q^{4} ; q^{4}\right)_{\infty}}\left[q^{16},-q^{6},-q^{10} ; q^{16}\right]_{\infty} \\
& \left(-q ; q^{2}\right)_{\infty}-\left(q ; q^{2}\right)_{\infty}=\frac{2 q}{\left(q^{4} ; q^{4}\right)_{\infty}}\left[q^{16},-q^{2},-q^{14} ; q^{16}\right]_{\infty} \\
& \left(-q ; q^{2}\right)_{\infty}^{2}+\left(q ; q^{2}\right)_{\infty}^{2}=2\left(-q^{2} ; q^{2}\right)_{\infty} \frac{\left(-q^{4} ; q^{8}\right)_{\infty}^{2}}{\left(q^{4} ; q^{8}\right)_{\infty}} \\
& \left(-q ; q^{2}\right)_{\infty}^{2}-\left(q ; q^{2}\right)_{\infty}^{2}=4 q\left(-q^{2} ; q^{2}\right)_{\infty} \frac{\left(-q^{8} ; q^{8}\right)_{\infty}^{2}}{\left(q^{4} ; q^{8}\right)_{\infty}} \\
& \left(-q ; q^{2}\right)_{\infty}^{4}+\left(q ; q^{2}\right)_{\infty}^{4}=2 \frac{\left(-q^{2} ; q^{4}\right)_{\infty}^{4}}{\left(q^{2} ; q^{4}\right)_{\infty}^{2}} \\
& \left(-q ; q^{2}\right)_{\infty}^{4}-\left(q ; q^{2}\right)_{\infty}^{4}=8 q \frac{\left(-q^{4} ; q^{4}\right)_{\infty}^{4}}{\left(q^{2} ; q^{4}\right)_{\infty}^{2}} \\
& \left(-q ; q^{2}\right)_{\infty}^{8}-\left(q ; q^{2}\right)_{\infty}^{8}=16 q\left(-q^{2} ; q^{2}\right)_{\infty}^{8} .
\end{aligned}
$$

For more information, the reader can consult [8, Eq. 2.1.4] for (3.1c), [23, p. 51] for (3.1d) and (3.1f), [8, Eq. 2.1.6] for (3.1e) and [8, Eqs. 2.1.10 \& 3.1.6], [15, Eq. 1], [23, Eq. 9] and [25, p. 470] for (3.1g).

Proof. Rewriting the six differences

$$
\begin{aligned}
\left(-q ; q^{2}\right)_{\infty} \pm\left(q ; q^{2}\right)_{\infty} & =\left\langle-q ; q^{4}\right\rangle_{\infty} \pm\left\langle q ; q^{4}\right\rangle_{\infty} \\
& =\left\langle-q,-q^{5} ; q^{8}\right\rangle_{\infty} \pm\left\langle q, q^{5} ; q^{8}\right\rangle_{\infty} \\
\left(-q ; q^{2}\right)_{\infty}^{2} \pm\left(q ; q^{2}\right)_{\infty}^{2} & =\left\langle-q,-q ; q^{4}\right\rangle_{\infty} \pm\left\langle q, q ; q^{4}\right\rangle_{\infty} \\
\left(-q ; q^{2}\right)_{\infty}^{4} \pm\left(q ; q^{2}\right)_{\infty}^{4} & =\left\langle-q,-q ; q^{2}\right\rangle_{\infty} \pm\left\langle q, q ; q^{2}\right\rangle_{\infty}
\end{aligned}
$$

and applying Proposition 3, we derive the first six identities displayed in the example. The last one follows from the product of (3.1e) and (3.1f).

$\S 3.2$. Identities modulo 4 and 8 . We show now a few identities modulo 4 and 8 . 
ExAmPle 2 (See [8, Eq. 2.1.8] and Ewell [14, Eq. 2.3] for the third identity).

$$
\begin{aligned}
& \frac{\left(-q ; q^{2}\right)_{\infty}}{\left(q ; q^{2}\right)_{\infty}}+\frac{\left(-q^{2} ; q^{4}\right)_{\infty}^{2}}{\left(q^{2} ; q^{4}\right)_{\infty}^{2}}=\frac{2\left(-q^{4} ; q^{4}\right)_{\infty}^{2}}{\left(q^{2} ; q^{4}\right)_{\infty}^{2}} \frac{\left[q^{3}, q^{5} ; q^{8}\right]_{\infty}^{2}}{\left(q ; q^{2}\right)_{\infty}} \\
& \frac{\left(-q ; q^{2}\right)_{\infty}}{\left(q ; q^{2}\right)_{\infty}}-\frac{\left(-q^{2} ; q^{4}\right)_{\infty}^{2}}{\left(q^{2} ; q^{4}\right)_{\infty}^{2}}=\frac{2 q\left(-q^{4} ; q^{4}\right)_{\infty}^{2}}{\left(q^{2} ; q^{4}\right)_{\infty}^{2}} \frac{\left[q, q^{7} ; q^{8}\right]_{\infty}^{2}}{\left(q ; q^{2}\right)_{\infty}} \\
& \frac{\left(-q ; q^{2}\right)_{\infty}^{2}}{\left(q ; q^{2}\right)_{\infty}^{2}}-\frac{\left(-q^{2} ; q^{4}\right)_{\infty}^{4}}{\left(q^{2} ; q^{4}\right)_{\infty}^{4}}=4 q \frac{\left(-q^{4} ; q^{4}\right)_{\infty}^{4}}{\left(q^{2} ; q^{4}\right)_{\infty}^{4}}
\end{aligned}
$$

Proof. Reformulating the differences

$$
\frac{\left(-q ; q^{2}\right)_{\infty}}{\left(q ; q^{2}\right)_{\infty}} \pm \frac{\left(-q^{2} ; q^{4}\right)_{\infty}^{2}}{\left(q^{2} ; q^{4}\right)_{\infty}^{2}}=\frac{\left\langle-q, q^{2} ; q^{4}\right\rangle_{\infty} \pm\left\langle q,-q^{2} ; q^{4}\right\rangle_{\infty}}{\left(q ; q^{2}\right)_{\infty}\left(q^{2} ; q^{4}\right)_{\infty}^{2}}
$$

and applying Proposition 3, we derive the first two identities displayed in the example. The third identity follows from the product of the first two.

EXAmPle 3 (See [8, Eq. 2.1.9] for the third identity).

$$
\begin{aligned}
& \frac{\left(-q ; q^{2}\right)_{\infty}}{\left(q ; q^{2}\right)_{\infty}}+\frac{\left(q^{2} ; q^{4}\right)_{\infty}^{2}}{\left(-q^{2} ; q^{4}\right)_{\infty}^{2}}=2 \frac{(-q ; q)_{\infty}}{\left(-q^{2} ; q^{4}\right)_{\infty}^{2}}\left[-q^{3},-q^{4},-q^{5},-q^{8} ; q^{8}\right]_{\infty}^{2} \\
& \frac{\left(-q ; q^{2}\right)_{\infty}}{\left(q ; q^{2}\right)_{\infty}}-\frac{\left(q^{2} ; q^{4}\right)_{\infty}^{2}}{\left(-q^{2} ; q^{4}\right)_{\infty}^{2}}=2 q \frac{(-q ; q)_{\infty}}{\left(-q^{2} ; q^{4}\right)_{\infty}^{2}}\left[-q,-q^{4},-q^{7},-q^{8} ; q^{8}\right]_{\infty}^{2} \\
& \frac{\left(-q ; q^{2}\right)_{\infty}^{2}}{\left(q ; q^{2}\right)_{\infty}^{2}}-\frac{\left(q^{2} ; q^{4}\right)_{\infty}^{4}}{\left(q^{2} ; q^{4}\right)_{\infty}^{4}}=4 q \frac{\left(-q ; q^{2}\right)_{\infty}^{2}}{\left(q ; q^{2}\right)_{\infty}^{2}} \frac{\left(-q^{4} ; q^{4}\right)_{\infty}^{4}}{\left(-q^{2} ; q^{4}\right)_{\infty}^{4}}
\end{aligned}
$$

Proof. Rewriting the differences

$$
\frac{(-q ; q)_{\infty}}{\left(-q^{2} ; q^{4}\right)_{\infty}^{2}}\left\{\left\langle-q,-q^{2} ; q^{4}\right\rangle_{\infty} \pm\left\langle q, q^{2} ; q^{4}\right\rangle_{\infty}\right\}
$$

and applying Proposition 3, we derive the first two identities displayed in the example. The third identity follows from the product of the first two.

Similarly, by means of Theorem 2, we can also derive the following identities.

$$
\begin{aligned}
& \left\langle-q,-q,-q,-q^{3} ; q^{8}\right\rangle_{\infty}+\left\langle q, q, q, q^{3} ; q^{8}\right\rangle_{\infty}=2 \frac{\left(-q^{2} ; q^{4}\right)_{\infty}^{3}}{\left(q^{8} ; q^{16}\right)_{\infty}^{2}} \\
& \left\langle-q,-q,-q^{2},-q^{4} ; q^{8}\right\rangle_{\infty}+\left\langle q, q, q^{2}, q^{4} ; q^{8}\right\rangle_{\infty}=2 \frac{\left[-q^{8},-q^{3},-q^{5} ; q^{8}\right]_{\infty}^{2}}{\left[q^{2},-q^{4} ; q^{4}\right]_{\infty}}
\end{aligned}
$$

EXAMPLE 4 (Theta function identity).

$$
\frac{\left(-q^{4} ; q^{8}\right)_{\infty}}{\left(-q^{8} ; q^{8}\right)_{\infty}}(-q ; q)_{\infty}-\frac{\left(q^{4} ; q^{8}\right)_{\infty}}{\left(q^{8} ; q^{8}\right)_{\infty}}(q ; q)_{\infty}=2 q \frac{\left(-q^{8} ; q^{8}\right)_{\infty}}{\left(-q^{4} ; q^{8}\right)_{\infty}}(-q ; q)_{\infty}
$$

It should be pointed out that the author has not been able to find the additive version for this last formula. 
Proof. Reformulating the difference

$$
\begin{aligned}
& \frac{\left(-q^{4} ; q^{8}\right)_{\infty}}{\left(-q^{8} ; q^{8}\right)_{\infty}}(-q ; q)_{\infty}-\frac{\left(q^{4} ; q^{8}\right)_{\infty}}{\left(q^{8} ; q^{8}\right)_{\infty}}(q ; q)_{\infty} \\
= & \left\langle-q,-q^{2},-q^{3},-q^{4} ; q^{8}\right\rangle_{\infty}-\left\langle q, q^{2}, q^{3}, q^{4} ; q^{8}\right\rangle_{\infty}
\end{aligned}
$$

and then applying Theorem 1, we derive the identity stated in the example.

Multiplying by $\frac{\left(q ; q^{2}\right)_{\infty}}{\left(-q^{4} ; q^{4}\right)_{\infty}}\left(q^{8} ; q^{8}\right)_{\infty}^{3}$ on both sides of the identity displayed in the last example, we recover an identity due to Berndt [3, Example on p. 119]:

$$
\phi^{2}\left(-q^{8}\right) f\left(-q^{8}\right)-\psi\left(q^{2}\right) f^{2}(-q)=2 q \psi\left(q^{8}\right) f^{2}\left(-q^{4}\right) .
$$

§3.3. Theta function Identities modulo 6. By means of modular equations, Kongsiriwong and Liu [19, Eqs. 8.22-8.23] found the following two identities:

$$
\begin{aligned}
& \psi(q) \psi\left(q^{3}\right)+\psi(-q) \psi\left(-q^{3}\right)=2 \phi\left(q^{6}\right) \psi\left(q^{4}\right) \\
& \psi(q) \psi\left(q^{3}\right)-\psi(-q) \psi\left(-q^{3}\right)=2 q \phi\left(q^{2}\right) \psi\left(q^{12}\right) .
\end{aligned}
$$

They may equivalently be stated in the following example.

EXAMPLE 5 (Kongsiriwong-Liu [19, Eqs. 8.22-8.23] for the first two identities).

$$
\begin{aligned}
& \frac{\left(-q ; q^{2}\right)_{\infty}}{\left(q ; q^{2}\right)_{\infty}}+\frac{\left(q^{3} ; q^{6}\right)_{\infty}}{\left(-q^{3} ; q^{6}\right)_{\infty}}=2 \frac{(-q ; q)_{\infty}}{\left(-q^{3} ; q^{6}\right)_{\infty}}\left(-q^{6} ; q^{6}\right)_{\infty}^{2}\left\langle-q^{4},-q^{4} ; q^{12}\right\rangle_{\infty} \\
& \frac{\left(-q ; q^{2}\right)_{\infty}}{\left(q ; q^{2}\right)_{\infty}}-\frac{\left(q^{3} ; q^{6}\right)_{\infty}}{\left(-q^{3} ; q^{6}\right)_{\infty}}=2 q \frac{(-q ; q)_{\infty}}{\left(-q^{3} ; q^{6}\right)_{\infty}}\left(-q^{6} ; q^{6}\right)_{\infty}^{2}\left\langle-q^{2},-q^{2} ; q^{12}\right\rangle_{\infty} \\
& \frac{\left(-q ; q^{2}\right)_{\infty}^{2}}{\left(q ; q^{2}\right)_{\infty}^{2}}-\frac{\left(q^{3} ; q^{6}\right)_{\infty}^{2}}{\left(-q^{3} ; q^{6}\right)_{\infty}^{2}}=4 q \frac{\left(-q^{2} ; q^{2}\right)_{\infty}^{2}}{\left(q ; q^{2}\right)_{\infty}^{2}} \frac{\left(-q^{6} ; q^{6}\right)_{\infty}^{2}}{\left(-q^{3} ; q^{6}\right)_{\infty}^{2}}
\end{aligned}
$$

The last identity follows from the product of the first two and corrects errors appear in [15, Eq. 2] where all the exponents of shifted factorials should be 2 instead of 4. One can find this correct form also in [8, Thm. 4.1] and Warnaar [23, Eq. 10].

Proof. Reformulating the differences

$$
\begin{aligned}
\frac{\left(-q ; q^{2}\right)_{\infty}}{\left(q ; q^{2}\right)_{\infty}} \pm \frac{\left(q^{3} ; q^{6}\right)_{\infty}}{\left(-q^{3} ; q^{6}\right)_{\infty}} & =\frac{(-q ; q)_{\infty}}{\left(-q^{3} ; q^{6}\right)_{\infty}}\left\{\left(-q ; q^{2}\right)_{\infty}\left(-q^{3} ; q^{6}\right)_{\infty} \pm\left(q ; q^{2}\right)_{\infty}\left(q^{3} ; q^{6}\right)_{\infty}\right\} \\
& =\frac{(-q ; q)_{\infty}}{\left(-q^{3} ; q^{6}\right)_{\infty}}\left\{\left\langle-q,-q^{3} ; q^{6}\right\rangle_{\infty} \pm\left\langle q, q^{3} ; q^{6}\right\rangle_{\infty}\right\}
\end{aligned}
$$

and then applying Proposition 3, we get two identities stated in the example.

Multiplying by $\left[q^{2}, q, q ; q^{2}\right]_{\infty} \times\left[q^{6},-q^{3},-q^{3} ; q^{6}\right]_{\infty}$ on both sides of the identity $(3.7 \mathrm{c})$, the resulting identity reads as

$$
\phi(q) \phi\left(q^{3}\right)-\phi(-q) \phi\left(-q^{3}\right)=4 q \psi\left(q^{2}\right) \psi\left(q^{6}\right) .
$$


EXAMPLE 6 (Shen [22, Eq. 4.7]).

$$
1+q \frac{\left(-q^{3} ; q^{3}\right)_{\infty}^{9}}{(-q ; q)_{\infty}^{3}}=\frac{\left(-q^{3} ; q^{3}\right)_{\infty}\left(q^{2} ; q^{2}\right)_{\infty}^{4}}{\left(q ; q^{2}\right)_{\infty}\left(q^{3} ; q^{3}\right)_{\infty}^{4}}
$$

Proof. Rewriting the difference and then applying Theorem 1, we get

$$
\begin{aligned}
1-\frac{\left(-q^{3} ; q^{3}\right)_{\infty}\left(q^{2} ; q^{2}\right)_{\infty}^{4}}{\left(q ; q^{2}\right)_{\infty}\left(q^{3} ; q^{3}\right)_{\infty}^{4}} & =1-\frac{\left\langle q^{2} ; q^{6}\right\rangle_{\infty}^{4}}{\left\langle q, q^{3}, q^{3}, q^{3} ; q^{6}\right\rangle_{\infty}} \\
& =\frac{\left\langle q^{5}, q^{3}, q^{3}, q^{3} ; q^{6}\right\rangle_{\infty}-\left\langle q^{2}, q^{4}, q^{4}, q^{4} ; q^{6}\right\rangle_{\infty}}{\left\langle q, q^{3}, q^{3}, q^{3} ; q^{6}\right\rangle_{\infty}} \\
& =-q \frac{\left\langle q ; q^{6}\right\rangle_{\infty}^{3}}{\left\langle q^{3} ; q^{6}\right\rangle_{\infty}^{3}}=-q \frac{\left(q ; q^{2}\right)_{\infty}^{3}}{\left(q^{3} ; q^{6}\right)_{\infty}^{9}}=-q \frac{\left(-q^{3} ; q^{3}\right)_{\infty}^{9}}{(-q ; q)_{\infty}^{3}}
\end{aligned}
$$

This is equivalent to the formula stated in the example.

EXAMPLE 7 (Dual formulae of Example 5).

$$
\begin{aligned}
& \frac{\left(-q ; q^{2}\right)_{\infty}}{\left(q ; q^{2}\right)_{\infty}}+\frac{\left(-q^{3} ; q^{6}\right)_{\infty}}{\left(q^{3} ; q^{6}\right)_{\infty}}=2 \frac{\left(-q^{3} ; q^{6}\right)_{\infty}}{\left(q ; q^{2}\right)_{\infty}}\left(-q^{12} ; q^{12}\right)_{\infty}^{2}\left\langle-q^{6},-q^{8} ; q^{24}\right\rangle_{\infty} \\
& \frac{\left(-q ; q^{2}\right)_{\infty}}{\left(q ; q^{2}\right)_{\infty}}-\frac{\left(-q^{3} ; q^{6}\right)_{\infty}}{\left(q^{3} ; q^{6}\right)_{\infty}}=2 q \frac{\left(-q^{3} ; q^{6}\right)_{\infty}}{\left(q ; q^{2}\right)_{\infty}}\left(-q^{12} ; q^{12}\right)_{\infty}^{2}\left\langle-q^{4},-q^{6} ; q^{24}\right\rangle_{\infty} \\
& \frac{\left(-q ; q^{2}\right)_{\infty}^{2}}{\left(q ; q^{2}\right)_{\infty}^{2}}+\frac{\left(-q^{3} ; q^{6}\right)_{\infty}^{2}}{\left(q^{3} ; q^{6}\right)_{\infty}^{2}}=2 \frac{\left(-q^{3} ; q^{3}\right)_{\infty}^{2}}{\left(q ; q^{2}\right)_{\infty}^{2}}\left(-q^{2} ; q^{4}\right)_{\infty}\left(-q^{6} ; q^{12}\right)_{\infty} \\
& \frac{\left(-q ; q^{2}\right)_{\infty}^{2}}{\left(q ; q^{2}\right)_{\infty}^{2}}-\frac{\left(-q^{3} ; q^{6}\right)_{\infty}^{2}}{\left(q^{3} ; q^{6}\right)_{\infty}^{2}}=4 q \frac{\left(-q^{3} ; q^{3}\right)_{\infty}^{2}}{\left(q ; q^{2}\right)_{\infty}^{2}}\left(-q^{4} ; q^{4}\right)_{\infty}\left(-q^{12} ; q^{12}\right)_{\infty} \\
& \frac{\left(-q ; q^{2}\right)_{\infty}^{4}}{\left(q ; q^{2}\right)_{\infty}^{4}}-\frac{\left(-q^{3} ; q^{6}\right)_{\infty}^{4}}{\left(q^{3} ; q^{6}\right)_{\infty}^{4}}=8 q \frac{\left(-q^{3} ; q^{3}\right)_{\infty}^{4}}{\left(q ; q^{2}\right)_{\infty}^{4}} \frac{\left(-q^{6} ; q^{6}\right)_{\infty}}{\left(q^{2} ; q^{4}\right)_{\infty}} .
\end{aligned}
$$

Proof. Reformulating the four differences

$$
\begin{aligned}
& \frac{\left(-q ; q^{2}\right)_{\infty}}{\left(q ; q^{2}\right)_{\infty}} \pm \frac{\left(-q^{3} ; q^{6}\right)_{\infty}}{\left(q^{3} ; q^{6}\right)_{\infty}}=\frac{\left(-q^{3} ; q^{6}\right)_{\infty}}{\left(q ; q^{2}\right)_{\infty}}\left\{\left\langle-q,-q^{7} ; q^{12}\right\rangle_{\infty} \pm\left\langle q, q^{7} ; q^{12}\right\rangle_{\infty}\right\} \\
& \frac{\left(-q ; q^{2}\right)_{\infty}^{2}}{\left(q ; q^{2}\right)_{\infty}^{2}} \pm \frac{\left(-q^{3} ; q^{6}\right)_{\infty}^{2}}{\left(q^{3} ; q^{6}\right)_{\infty}^{2}}=\frac{\left(-q^{3} ; q^{6}\right)_{\infty}^{2}}{\left(q ; q^{2}\right)_{\infty}^{2}}\left\{\left\langle-q,-q ; q^{6}\right\rangle_{\infty} \pm\left\langle q, q ; q^{6}\right\rangle_{\infty}\right\}
\end{aligned}
$$

and then applying Proposition 3, we derive the first four identities displayed in the example. The last identity (3.9e) follows from the product of (3.9c) and (3.9d).

EXAMPLE 8 (Three theta function identities).

$$
\begin{aligned}
& \frac{\left(-q ; q^{2}\right)_{\infty}}{\left(q ; q^{2}\right)_{\infty}}+\frac{\left(-q^{3} ; q^{6}\right)_{\infty}^{3}}{\left(q^{3} ; q^{6}\right)_{\infty}^{3}}=2 \frac{(-q ; q)_{\infty}\left(-q^{6} ; q^{6}\right)_{\infty}}{\left(q^{3} ; q^{6}\right)_{\infty}^{3}} \frac{\left(q^{4} ; q^{4}\right)_{\infty}^{2}}{\left(q^{12} ; q^{12}\right)_{\infty}^{2}} \\
& \frac{\left(-q ; q^{2}\right)_{\infty}}{\left(q ; q^{2}\right)_{\infty}}-\frac{\left(-q^{3} ; q^{6}\right)_{\infty}^{3}}{\left(q^{3} ; q^{6}\right)_{\infty}^{3}}=2 q \frac{(-q ; q)_{\infty}\left(-q^{6} ; q^{6}\right)_{\infty}}{\left(q^{3} ; q^{6}\right)_{\infty}^{3}} \frac{\left(q^{2} ; q^{4}\right)_{\infty}^{2}}{\left(q^{6} ; q^{12}\right)_{\infty}^{2}} \\
& \frac{\left(-q ; q^{2}\right)_{\infty}^{2}}{\left(q ; q^{2}\right)_{\infty}^{2}}-\frac{\left(-q^{3} ; q^{6}\right)_{\infty}^{6}}{\left(q^{3} ; q^{6}\right)_{\infty}^{6}}=4 q \frac{(-q ; q)_{\infty}^{2}\left(-q^{6} ; q^{6}\right)_{\infty}^{2}}{\left(q^{3} ; q^{6}\right)_{\infty}^{4}} \frac{\left(q^{2} ; q^{2}\right)_{\infty}^{2}}{\left(q^{3} ; q^{3}\right)_{\infty}^{2}}
\end{aligned}
$$


Proof. Rewrite the differences as follows:

$$
\frac{\left(-q ; q^{2}\right)_{\infty}}{\left(q ; q^{2}\right)_{\infty}} \pm \frac{\left(-q^{3} ; q^{6}\right)_{\infty}^{3}}{\left(q^{3} ; q^{6}\right)_{\infty}^{3}}=\frac{(-q ; q)_{\infty}\left(-q^{3} ; q^{6}\right)_{\infty}}{\left(q^{3} ; q^{6}\right)_{\infty}^{2}}\left\{\left\langle-q, q^{3} ; q^{6}\right\rangle_{\infty} \pm\left\langle q,-q^{3} ; q^{6}\right\rangle_{\infty}\right\}
$$

Applying Proposition 3 and then simplifying the result, we get the first two identities stated in the example. The last one follows from the product of the first two identities.

EXAMPLE 9 (Three theta function identities).

$$
\begin{aligned}
& \frac{\left(-q ; q^{2}\right)_{\infty}^{3}}{\left(q ; q^{2}\right)_{\infty}^{3}}+\frac{\left(-q^{3} ; q^{6}\right)_{\infty}}{\left(q^{3} ; q^{6}\right)_{\infty}}=2 \frac{\left(-q^{2} ; q^{2}\right)_{\infty}^{3}}{\left(q ; q^{2}\right)_{\infty}^{3}} \frac{\left(-q^{3} ; q^{6}\right)_{\infty}}{\left(-q^{6} ; q^{6}\right)_{\infty}} \\
& \frac{\left(-q ; q^{2}\right)_{\infty}^{3}}{\left(q ; q^{2}\right)_{\infty}^{3}}-\frac{\left(-q^{3} ; q^{6}\right)_{\infty}}{\left(q^{3} ; q^{6}\right)_{\infty}}=6 q \frac{\left(-q ; q^{2}\right)_{\infty}}{(q ; q)_{\infty}^{2}} \frac{\left(q^{12} ; q^{12}\right)_{\infty}^{2}}{\left(q^{3} ; q^{6}\right)_{\infty}} \\
& \frac{\left(-q ; q^{2}\right)_{\infty}^{6}}{\left(q ; q^{2}\right)_{\infty}^{6}}-\frac{\left(-q^{3} ; q^{6}\right)_{\infty}^{2}}{\left(q^{3} ; q^{6}\right)_{\infty}^{2}}=12 q \frac{(-q ; q)_{\infty}^{2}\left(-q^{2} ; q^{2}\right)_{\infty}^{2}}{(q ; q)_{\infty}^{2}\left(q ; q^{2}\right)_{\infty}^{2}} \frac{\left(q^{6} ; q^{6}\right)_{\infty}^{2}}{\left(q^{3} ; q^{6}\right)_{\infty}^{2}}
\end{aligned}
$$

Proof. Let $\omega=\exp (2 \pi i / 3)$, a cube root of unity. Reformulate the differences as follows:

$$
\frac{\left(-q ; q^{2}\right)_{\infty}^{3}}{\left(q ; q^{2}\right)_{\infty}^{3}} \pm \frac{\left(-q^{3} ; q^{6}\right)_{\infty}}{\left(q^{3} ; q^{6}\right)_{\infty}}=\frac{\left(q^{2} ; q^{4}\right)_{\infty}}{\left(q ; q^{2}\right)_{\infty}^{3}\left(q^{3} ; q^{6}\right)_{\infty}}\left\{\left\langle-q, q \omega ; q^{2}\right\rangle_{\infty} \pm\left\langle q,-q \omega ; q^{2}\right\rangle_{\infty}\right\}
$$

Applying Proposition 3 and then simplifying the results, we get the first two identities stated in the example. The third one follows from the product of the first two, which seems to be a very unusual theta function identity.

Multiplying both sides at (3.11a) and (3.11b) by $(q ; q)_{\infty}^{3} \frac{\left(-q^{6} ; q^{6}\right)_{\infty}}{\left(q^{3} ; q^{3}\right)_{\infty}}$, we may state the resulting identities in terms of Ramanujan's functions;

$$
\begin{aligned}
& \frac{\phi^{3}\left(-q^{2}\right)}{\phi\left(-q^{6}\right)}+\frac{\phi^{3}(-q)}{\phi\left(-q^{3}\right)}=2 \frac{\psi^{3}(-q)}{\psi\left(-q^{3}\right)} \\
& \frac{\phi^{3}\left(-q^{2}\right)}{\phi\left(-q^{6}\right)}-\frac{\phi^{3}(-q)}{\phi\left(-q^{3}\right)}=6 q \phi(-q) \psi\left(q^{6}\right) \frac{\chi(-q) \chi\left(-q^{2}\right)}{\chi\left(-q^{3}\right) \chi\left(-q^{6}\right)} .
\end{aligned}
$$

Considering $3 \mathrm{Eq}(3.11 \mathrm{a})+\mathrm{Eq}(3.11 \mathrm{~b})$, we can factorize the right hand side into

$$
\begin{aligned}
6 & \left\{\frac{\left(-q^{2} ; q^{2}\right)_{\infty}^{3}}{\left(q ; q^{2}\right)_{\infty}^{3}} \frac{\left(-q^{3} ; q^{6}\right)_{\infty}}{\left(-q^{6} ; q^{6}\right)_{\infty}}+q \frac{\left(-q ; q^{2}\right)_{\infty}}{(q ; q)_{\infty}^{2}} \frac{\left(q^{12} ; q^{12}\right)_{\infty}^{2}}{\left(q^{3} ; q^{6}\right)_{\infty}}\right\} \\
& =6 \frac{\left(q^{4} ; q^{4}\right)_{\infty}\left(q^{6} ; q^{6}\right)_{\infty}^{2}}{(q ; q)_{\infty}^{3}\left(q^{3} ; q^{6}\right)_{\infty}}\left\{\left\langle q^{4}, q^{4} ; q^{12}\right\rangle_{\infty}+q\left\langle q^{2}, q^{2} ; q^{12}\right\rangle_{\infty}\right\} \\
& =6 \frac{\left(q^{4} ; q^{4}\right)_{\infty}\left(q^{6} ; q^{6}\right)_{\infty}^{2}}{(q ; q)_{\infty}^{3}\left(q^{3} ; q^{6}\right)_{\infty}} \frac{\left\langle-q, q^{3} ; q^{6}\right\rangle_{\infty}}{\left(-q^{6} ; q^{6}\right)_{\infty}^{2}}=6 \frac{(-q ; q)_{\infty}^{2}\left(q^{3} ; q^{3}\right)_{\infty}^{2}}{(q ; q)_{\infty}^{2}\left(-q^{6} ; q^{6}\right)_{\infty}}
\end{aligned}
$$

where the last line is derived from Corollary 4 under the replacements $u \rightarrow-q^{2}, v \rightarrow q$ and $q \rightarrow q^{6}$. Therefore we have established the following theta function identity. 
EXAMPLE 10 (Theta function identity).

$$
2 \frac{\left(-q ; q^{2}\right)_{\infty}^{3}}{\left(q ; q^{2}\right)_{\infty}^{3}}+\frac{\left(-q^{3} ; q^{6}\right)_{\infty}}{\left(q^{3} ; q^{6}\right)_{\infty}}=3 \frac{(-q ; q)_{\infty}^{2}\left(q^{3} ; q^{3}\right)_{\infty}^{2}}{(q ; q)_{\infty}^{2}\left(-q^{6} ; q^{6}\right)_{\infty}}
$$

In terms of Ramanujan's $\phi$-function, it reads as

$$
2 \frac{\phi^{3}\left(-q^{2}\right)}{\phi\left(-q^{6}\right)}+\frac{\phi^{3}(-q)}{\phi\left(-q^{3}\right)}=3 \phi(-q) \phi\left(-q^{3}\right) .
$$

$\S 3.4$. Theta function identities of Farkas-Kra type. Here we present theta function identities modulo 10, 14 and 18 of Farkas-Kra type.

EXAMPLE 11 (Three theta function identities).

$$
\begin{aligned}
& \frac{\left(-q ; q^{2}\right)_{\infty}}{\left(q ; q^{2}\right)_{\infty}}+\frac{\left(-q^{5} ; q^{10}\right)_{\infty}}{\left(q^{5} ; q^{10}\right)_{\infty}}=2 \frac{\left(-q^{4} ; q^{4}\right)_{\infty}}{\left(q ; q^{2}\right)_{\infty}} \frac{\left(-q^{10} ; q^{20}\right)_{\infty}}{\left(q^{5} ; q^{10}\right)_{\infty}} \\
& \frac{\left(-q ; q^{2}\right)_{\infty}}{\left(q ; q^{2}\right)_{\infty}}-\frac{\left(-q^{5} ; q^{10}\right)_{\infty}}{\left(q^{5} ; q^{10}\right)_{\infty}}=2 q \frac{\left(-q^{2} ; q^{4}\right)_{\infty}}{\left(q ; q^{2}\right)_{\infty}} \frac{\left(-q^{20} ; q^{20}\right)_{\infty}}{\left(q^{5} ; q^{10}\right)_{\infty}} \\
& \frac{\left(-q ; q^{2}\right)_{\infty}^{2}}{\left(q ; q^{2}\right)_{\infty}^{2}}+\frac{\left(-q^{5} ; q^{10}\right)_{\infty}^{2}}{\left(q^{5} ; q^{10}\right)_{\infty}^{2}}=4 q \frac{\left(-q^{2} ; q^{2}\right)_{\infty}}{\left(q ; q^{2}\right)_{\infty}^{2}} \frac{\left(-q^{10} ; q^{10}\right)_{\infty}}{\left(q^{5} ; q^{10}\right)_{\infty}^{2}}
\end{aligned}
$$

Proof. Reformulating the differences on the left hand sides as

$$
\begin{aligned}
\frac{\left(-q ; q^{2}\right)_{\infty}}{\left(q ; q^{2}\right)_{\infty}} \pm \frac{\left(-q^{5} ; q^{10}\right)_{\infty}}{\left(q^{5} ; q^{10}\right)_{\infty}} & =\frac{\left(-q^{5} ; q^{10}\right)_{\infty}}{\left(q ; q^{2}\right)_{\infty}}\left\{\frac{\left(-q ; q^{2}\right)_{\infty}}{\left(-q^{5} ; q^{10}\right)_{\infty}} \pm \frac{\left(q ; q^{2}\right)_{\infty}}{\left(q^{5} ; q^{10}\right)_{\infty}}\right\} \\
& =\frac{\left(-q^{5} ; q^{10}\right)_{\infty}}{\left(q ; q^{2}\right)_{\infty}}\left\{\left\langle-q,-q^{3} ; q^{10}\right\rangle_{\infty} \pm\left\langle q, q^{3} ; q^{10}\right\rangle_{\infty}\right\}
\end{aligned}
$$

and then applying Proposition 3, we derive the first two identities stated in the example. The third one follows from the product of the first two.

EXAMPLE 12 ([15, Eq. 3]: see also Warnaar [23, Eq. 1]).

$$
\frac{\left(-q ; q^{2}\right)_{\infty}}{\left(q ; q^{2}\right)_{\infty}}-\frac{\left(q^{7} ; q^{14}\right)_{\infty}}{\left(-q^{7} ; q^{14}\right)_{\infty}}=2 q \frac{\left(-q^{2} ; q^{2}\right)_{\infty}}{\left(q ; q^{2}\right)_{\infty}} \frac{\left(-q^{14} ; q^{14}\right)_{\infty}}{\left(-q^{7} ; q^{14}\right)_{\infty}}
$$

We point out that this identity has appeared earlier, but hidden in [8, p. 112(b)].

Proof. Rewriting the difference on the left hand side and then factorizing the numerator by means of Theorem 1 , we get

$$
\begin{aligned}
\frac{\left(-q ; q^{2}\right)_{\infty}}{\left(q ; q^{2}\right)_{\infty}}-\frac{\left(q^{7} ; q^{14}\right)_{\infty}}{\left(-q^{7} ; q^{14}\right)_{\infty}} & =\frac{\left(-q ; q^{2}\right)_{\infty}\left(-q^{7} ; q^{14}\right)_{\infty}-\left(q ; q^{2}\right)_{\infty}\left(q^{7} ; q^{14}\right)_{\infty}}{\left(q ; q^{2}\right)_{\infty}\left(-q^{7} ; q^{14}\right)_{\infty}} \\
& =\frac{\left\langle-q,-q^{3},-q^{5},-q^{7} ; q^{14}\right\rangle_{\infty}-\left\langle q, q^{3}, q^{5}, q^{7} ; q^{14}\right\rangle_{\infty}}{\left(q ; q^{2}\right)_{\infty}\left(-q^{7} ; q^{14}\right)_{\infty}} \\
& =\frac{q\left\langle-1,-q^{2},-q^{4},-q^{8} ; q^{14}\right\rangle_{\infty}}{\left(q ; q^{2}\right)_{\infty}\left(-q^{7} ; q^{14}\right)_{\infty}}
\end{aligned}
$$

After some routine simplification, we derive the identity stated in the example. 
Another similar result modulo 18 reads as follows.

EXAMPLE 13 (Theta function identity).

$$
\frac{\left(-q ; q^{2}\right)_{\infty}}{\left(q ; q^{2}\right)_{\infty}}-\frac{\left(-q^{9} ; q^{18}\right)_{\infty}}{\left(q^{9} ; q^{18}\right)_{\infty}}=2 q \frac{\left(-q^{2} ; q^{2}\right)_{\infty}}{\left(q ; q^{2}\right)_{\infty}} \frac{\left(-q^{9} ; q^{9}\right)_{\infty}\left(-q^{18} ; q^{18}\right)_{\infty}}{\left(-q^{6} ; q^{6}\right)_{\infty}}
$$

Proof. The difference on the left hand side can be factorized, by means of Theorem 1, as follows:

$$
\begin{aligned}
& \frac{\left(-q ; q^{2}\right)_{\infty}}{\left(q ; q^{2}\right)_{\infty}}-\frac{\left(-q^{9} ; q^{18}\right)_{\infty}}{\left(q^{9} ; q^{18}\right)_{\infty}}=\frac{\left(-q^{9} ; q^{18}\right)_{\infty}}{\left(q ; q^{2}\right)_{\infty}}\left\{\frac{\left(-q ; q^{2}\right)_{\infty}}{\left(-q^{9} ; q^{18}\right)_{\infty}}-\frac{\left(q ; q^{2}\right)_{\infty}}{\left(q^{9} ; q^{18}\right)_{\infty}}\right\} \\
& =\frac{\left(-q^{9} ; q^{18}\right)_{\infty}}{\left(q ; q^{2}\right)_{\infty}}\left\{\left\langle-q,-q^{3},-q^{5},-q^{7} ; q^{18}\right\rangle_{\infty}-\left\langle q, q^{3}, q^{5}, q^{7} ; q^{18}\right\rangle_{\infty}\right\} \\
& =q \frac{\left(-q^{9} ; q^{18}\right)_{\infty}}{\left(q ; q^{2}\right)_{\infty}}\left\langle-1,-q^{2},-q^{4},-q^{8} ; q^{18}\right\rangle_{\infty} .
\end{aligned}
$$

After some routine simplification, we derive the identity stated in the example.

$\S 3.5$. Identities due to Blecksmith-Brillhart-Gerst. Multiplying (3.1a) and (3.1b) by $(q ; q)_{\infty}$, we recover the following two equivalent identities due to Blecksmith et al. [7, Theorem 1 and Corollary 1],

$$
\begin{aligned}
& \frac{\left(q^{2} ; q^{2}\right)_{\infty}}{\left(-q^{2} ; q^{2}\right)_{\infty}}+\frac{(q ; q)_{\infty}}{(-q ; q)_{\infty}}=2 \frac{\left(q ; q^{2}\right)_{\infty}}{\left(-q^{2} ; q^{2}\right)_{\infty}}\left[q^{16},-q^{6},-q^{10} ; q^{16}\right]_{\infty} \\
& \frac{\left(q^{2} ; q^{2}\right)_{\infty}}{\left(-q^{2} ; q^{2}\right)_{\infty}}-\frac{(q ; q)_{\infty}}{(-q ; q)_{\infty}}=2 q \frac{\left(q ; q^{2}\right)_{\infty}}{\left(-q^{2} ; q^{2}\right)_{\infty}}\left[q^{16},-q^{2},-q^{14} ; q^{16}\right]_{\infty}
\end{aligned}
$$

EXAMPLE 14 (See [7, Theorem 2 and Corollary 2] for the first two identities).

$$
\begin{aligned}
& \frac{\left(q^{3} ; q^{3}\right)_{\infty}}{\left(-q^{3} ; q^{3}\right)_{\infty}}+\frac{(q ; q)_{\infty}}{(-q ; q)_{\infty}}=\frac{2}{(-q ; q)_{\infty}}\left[q^{12},-q^{5},-q^{7} ; q^{12}\right]_{\infty} \\
& \frac{\left(q^{3} ; q^{3}\right)_{\infty}}{\left(-q^{3} ; q^{3}\right)_{\infty}}-\frac{(q ; q)_{\infty}}{(-q ; q)_{\infty}}=\frac{2 q}{(-q ; q)_{\infty}}\left[q^{12},-q,-q^{11} ; q^{12}\right]_{\infty} \\
& \frac{\left(q^{3} ; q^{3}\right)_{\infty}^{2}}{\left(-q^{3} ; q^{3}\right)_{\infty}^{2}}+\frac{(q ; q)_{\infty}^{2}}{(-q ; q)_{\infty}^{2}}=2\left(q^{6} ; q^{6}\right)_{\infty}^{2} \frac{\left(-q^{2} ; q^{2}\right)_{\infty}}{(-q ; q)_{\infty}^{2}} \frac{\left(-q^{3} ; q^{6}\right)_{\infty}^{2}}{\left(-q^{6} ; q^{6}\right)_{\infty}} \\
& \frac{\left(q^{3} ; q^{3}\right)_{\infty}^{2}}{\left(-q^{3} ; q^{3}\right)_{\infty}^{2}}-\frac{(q ; q)_{\infty}^{2}}{(-q ; q)_{\infty}^{2}}=4 q\left(q^{6} ; q^{6}\right)_{\infty}^{2} \frac{\left(-q ; q^{2}\right)_{\infty}}{(-q ; q)_{\infty}^{2}} \frac{\left(-q^{6} ; q^{6}\right)_{\infty}^{2}}{\left(-q^{3} ; q^{6}\right)_{\infty}} \\
& \frac{\left(q^{3} ; q^{3}\right)_{\infty}^{4}}{\left(-q^{3} ; q^{3}\right)_{\infty}^{4}}-\frac{(q ; q)_{\infty}^{4}}{(-q ; q)_{\infty}^{4}}=8 q\left(q^{6} ; q^{6}\right)_{\infty}^{4} \frac{\left(-q^{3} ; q^{3}\right)_{\infty}}{(-q ; q)_{\infty}^{3}}
\end{aligned}
$$


Proof. Reformulating the differences

$$
\begin{aligned}
\frac{\left(q^{3} ; q^{3}\right)_{\infty}}{\left(-q^{3} ; q^{3}\right)_{\infty}} \pm \frac{(q ; q)_{\infty}}{(-q ; q)_{\infty}} & =\frac{\left(q^{3} ; q^{3}\right)_{\infty}}{(-q ; q)_{\infty}}\left\{\left[-q,-q^{2} ; q^{3}\right]_{\infty} \pm\left[q, q^{2} ; q^{3}\right]_{\infty}\right\} \\
\frac{\left(q^{3} ; q^{3}\right)_{\infty}^{2}}{\left(-q^{3} ; q^{3}\right)_{\infty}^{2}} \pm \frac{(q ; q)_{\infty}^{2}}{(-q ; q)_{\infty}^{2}} & =\frac{\left(q^{3} ; q^{3}\right)_{\infty}^{2}}{(-q ; q)_{\infty}^{2}}\left\{\frac{(-q ; q)_{\infty}^{2}}{\left(-q^{3} ; q^{3}\right)_{\infty}^{2}} \pm \frac{(q ; q)_{\infty}^{2}}{\left(q^{3} ; q^{3}\right)_{\infty}^{2}}\right\} \\
& =\frac{\left(q^{3} ; q^{3}\right)_{\infty}^{2}}{(-q ; q)_{\infty}^{2}}\left\{\left\langle-q ; q^{3}\right\rangle_{\infty}^{2} \pm\left\langle q ; q^{3}\right\rangle_{\infty}^{2}\right\}
\end{aligned}
$$

and then applying Proposition 3, we get the first four identities stated in the example. The last one follows from the product of (3.16c) and (3.16d).

$\S$ 3.6. Theta function identities modulo 5 and 9 . We prove a new identity modulo 5 and three identities modulo 9 originally due to Shen [22].

EXAMPLE 15 (Three theta function identities).

$$
\begin{aligned}
& \frac{\left(q^{5} ; q^{5}\right)_{\infty}}{\left(-q^{5} ; q^{5}\right)_{\infty}}+\frac{(q ; q)_{\infty}}{(-q ; q)_{\infty}}=2 \frac{\left(q^{5} ; q^{5}\right)_{\infty}}{(-q ; q)_{\infty}}\left(-q^{5} ; q^{5}\right)_{\infty}^{2}\left\langle-q^{3},-q^{4} ; q^{10}\right\rangle_{\infty} \\
& \frac{\left(q^{5} ; q^{5}\right)_{\infty}}{\left(-q^{5} ; q^{5}\right)_{\infty}}-\frac{(q ; q)_{\infty}}{(-q ; q)_{\infty}}=2 q \frac{\left(q^{5} ; q^{5}\right)_{\infty}}{(-q ; q)_{\infty}}\left(-q^{5} ; q^{5}\right)_{\infty}^{2}\left\langle-q,-q^{2} ; q^{10}\right\rangle_{\infty} \\
& \frac{\left(q^{5} ; q^{5}\right)_{\infty}^{2}}{\left(-q^{5} ; q^{5}\right)_{\infty}^{2}}-\frac{(q ; q)_{\infty}^{2}}{(-q ; q)_{\infty}^{2}}=4 q \frac{\left(-q^{5} ; q^{5}\right)_{\infty}}{(-q ; q)_{\infty}}\left(q^{10} ; q^{10}\right)_{\infty}^{2} .
\end{aligned}
$$

Proof. Reformulating the differences

$$
\begin{aligned}
\frac{\left(q^{5} ; q^{5}\right)_{\infty}}{\left(-q^{5} ; q^{5}\right)_{\infty}} \pm \frac{(q ; q)_{\infty}}{(-q ; q)_{\infty}} & =\frac{\left(q^{5} ; q^{5}\right)_{\infty}}{(-q ; q)_{\infty}}\left\{\frac{(-q ; q)_{\infty}}{\left(-q^{5} ; q^{5}\right)_{\infty}} \pm \frac{(q ; q)_{\infty}}{\left(q^{5} ; q^{5}\right)_{\infty}}\right\} \\
& =\frac{\left(q^{5} ; q^{5}\right)_{\infty}}{(-q ; q)_{\infty}}\left\{\left\langle-q,-q^{2} ; q^{5}\right\rangle_{\infty} \pm\left\langle q, q^{2} ; q^{5}\right\rangle_{\infty}\right\}
\end{aligned}
$$

and then applying Proposition 3, we get the first two identities stated in the example, whose product results in the third one.

EXAMPLE 16 ([9, Eq. 2.16]: see also [22, Eq. 4.2]).

$$
\frac{\left(q^{9} ; q^{9}\right)_{\infty}}{\left(-q^{9} ; q^{9}\right)_{\infty}}-\frac{(q ; q)_{\infty}}{(-q ; q)_{\infty}}=2 q\left[q^{18}, q^{3}, q^{15} ; q^{18}\right]_{\infty}
$$

The author has not been able to find the additive version for this formula. 
Proof. The identity can be shown as follows:

$$
\begin{aligned}
\frac{\left(q^{9} ; q^{9}\right)_{\infty}}{\left(-q^{9} ; q^{9}\right)_{\infty}}-\frac{(q ; q)_{\infty}}{(-q ; q)_{\infty}} & =\frac{\left(q^{9} ; q^{9}\right)_{\infty}}{(-q ; q)_{\infty}}\left\{\frac{(-q ; q)_{\infty}}{\left(-q^{9} ; q^{9}\right)_{\infty}}-\frac{(q ; q)_{\infty}}{\left(q^{9} ; q^{9}\right)_{\infty}}\right\} \\
& =\frac{\left(q^{9} ; q^{9}\right)_{\infty}}{(-q ; q)_{\infty}}\left\{\begin{array}{r}
\left\langle-q,-q^{2},-q^{3},-q^{4} ; q^{9}\right\rangle_{\infty} \\
-\left\langle q, q^{2}, q^{3}, q^{4} ; q^{9}\right\rangle_{\infty}
\end{array}\right\} \\
& =q \frac{\left(q^{9} ; q^{9}\right)_{\infty}}{(-q ; q)_{\infty}}\left\langle-1,-q,-q^{2},-q^{5} ; q^{9}\right\rangle_{\infty} \\
& =2 q\left[q^{18}, q^{3}, q^{15} ; q^{18}\right]_{\infty},
\end{aligned}
$$

where the last equality has been justified by (1.1) under $a=-q^{5}, b=q, c=q^{2}, d=q^{3}$ and $e=q^{4}$.

Observe the equivalent products

$$
\left[q^{18}, q^{3}, q^{15} ; q^{18}\right]_{\infty}=\left(q^{18} ; q^{18}\right)_{\infty} \frac{\left(-q^{9} ; q^{9}\right)_{\infty}}{\left(-q^{3} ; q^{3}\right)_{\infty}} .
$$

Comparing the cubic form of Example 16 with the case $q \rightarrow q^{3}$ of (3.16e), we further recover the following identity.

EXAMPle 17 (Shen [22, Eq. 4.10]: cf. [3, Eqs. 24.28-24.29] and [8, Eq. 4.7.1]).

$$
\left\{\frac{\left(q^{9} ; q^{9}\right)_{\infty}}{\left(-q^{9} ; q^{9}\right)_{\infty}}-\frac{(q ; q)_{\infty}}{(-q ; q)_{\infty}}\right\}^{3}=\frac{\left(-q^{9} ; q^{9}\right)_{\infty}}{\left(q^{9} ; q^{9}\right)_{\infty}}\left\{\frac{\left(q^{9} ; q^{9}\right)_{\infty}^{4}}{\left(-q^{9} ; q^{9}\right)_{\infty}^{4}}-\frac{\left(q^{3} ; q^{3}\right)_{\infty}^{4}}{\left(-q^{3} ; q^{3}\right)_{\infty}^{4}}\right\}
$$

In terms of Ramanujan's functions, this may be restated as

$$
\phi^{4}\left(-q^{9}\right)-\phi^{4}\left(-q^{3}\right)=\phi\left(-q^{9}\right)\left\{\phi\left(-q^{9}\right)-\phi(-q)\right\}^{3} .
$$

We may further consider the difference,

$$
\begin{aligned}
3 \frac{\left(q^{9} ; q^{9}\right)_{\infty}}{\left(-q^{9} ; q^{9}\right)_{\infty}}-\frac{(q ; q)_{\infty}}{(-q ; q)_{\infty}} & =2 \frac{\left(q^{9} ; q^{9}\right)_{\infty}}{\left(-q^{9} ; q^{9}\right)_{\infty}}+2 q\left[q^{18}, q^{3}, q^{15} ; q^{18}\right]_{\infty} \\
& =2\left[q^{18}, q^{9}, q^{9} ; q^{18}\right]_{\infty}+2 q\left[q^{18}, q^{3}, q^{15} ; q^{18}\right]_{\infty} \\
& =2 \sum_{n}(-1)^{n} q^{18\left(\begin{array}{c}
n \\
2
\end{array}\right)+9 n}\left\{1+q^{1+6 n}\right\} \\
& =2\left[q^{6},-q,-q^{5} ; q^{6}\right]_{\infty}\left[q^{8}, q^{4} ; q^{12}\right]_{\infty} \\
& =2 \frac{(-q ; q)_{\infty}}{\left(-q^{3} ; q^{3}\right)_{\infty}}\left(q^{2} ; q^{2}\right)_{\infty},
\end{aligned}
$$

where we have appealed to the quintuple product identity (cf. [16, Ex, 5.6] and Gordon [17], for example):

$$
[q, z, q / z ; q]_{\infty}\left[q z^{2}, q / z^{2} ; q^{2}\right]_{\infty}=\sum_{n=-\infty}^{+\infty}\left\{1-z^{1+6 n}\right\} q^{3\left(\begin{array}{c}
n \\
2
\end{array}\right)\left(q^{2} / z^{3}\right)^{n}}
$$

This leads us to the following result. 
EXAMPLE 18 (Shen [22, Eq. 4.3]).

$$
3 \frac{\left(q^{9} ; q^{9}\right)_{\infty}}{\left(-q^{9} ; q^{9}\right)_{\infty}}-\frac{(q ; q)_{\infty}}{(-q ; q)_{\infty}}=2 \frac{(-q ; q)_{\infty}\left(q^{2} ; q^{2}\right)_{\infty}}{\left(-q^{3} ; q^{3}\right)_{\infty}}
$$

$\S 3.7$. Ramanujan's partition functions $G$ and $H$. We can also prove some identities on Ramanujan's partition functions $G$ and $H$, including three among the forty identities for the Rogers-Ramanujan functions (cf. [4] and [6]).

EXAMPLE 19 (Rogers [21, Eqs. 5-6] for the first two identities).

$$
\begin{aligned}
G(q)+G(-q) & =2 \frac{\left(q^{40} ; q^{40}\right)_{\infty}\left\langle-q^{12} ; q^{40}\right\rangle_{\infty}}{\left(q^{10} ; q^{10}\right)_{\infty}\left\langle q,-q, q^{6} ; q^{10}\right\rangle_{\infty}} \\
G(q)-G(-q) & =2 q \frac{\left(q^{40} ; q^{40}\right)_{\infty}\left\langle-q^{8} ; q^{40}\right\rangle_{\infty}}{\left(q^{10} ; q^{10}\right)_{\infty}\left\langle q,-q, q^{6} ; q^{10}\right\rangle_{\infty}} \\
G^{2}(q)-G^{2}(-q) & =4 q \frac{\left(q^{8} ; q^{8}\right)_{\infty}}{\left(q^{2} ; q^{2}\right)_{\infty}^{2}}\left[q^{40}, q^{12}, q^{28} ; q^{40}\right]_{\infty} .
\end{aligned}
$$

Proof. Rewriting

$$
G(q) \pm G(-q)=\frac{\left\langle-q ; q^{10}\right\rangle_{\infty} \pm\left\langle q ; q^{10}\right\rangle_{\infty}}{\left\langle q,-q, q^{6} ; q^{10}\right\rangle_{\infty}}=\frac{\left\langle-q,-q^{11} ; q^{20}\right\rangle_{\infty} \pm\left\langle q, q^{11} ; q^{20}\right\rangle_{\infty}}{\left\langle q,-q, q^{6} ; q^{10}\right\rangle_{\infty}}
$$

and then applying Proposition 3, we derive the first two identities stated in the example. The third one follows from the product of the first two.

EXAMPLE 20 (Rogers [21, Eqs. 7-8]).

$$
\begin{aligned}
H(q)+H(-q) & =2 \frac{\left(q^{40} ; q^{40}\right)_{\infty}\left\langle-q^{16} ; q^{40}\right\rangle_{\infty}}{\left(q^{10} ; q^{10}\right)_{\infty}\left\langle q^{2}, q^{7},-q^{7} ; q^{10}\right\rangle_{\infty}} \\
H(q)-H(-q) & =2 q^{3} \frac{\left(q^{40} ; q^{40}\right)_{\infty}\left\langle-q^{4} ; q^{40}\right\rangle_{\infty}}{\left(q^{10} ; q^{10}\right)_{\infty}\left\langle q^{2}, q^{7},-q^{7} ; q^{10}\right\rangle_{\infty}} \\
H^{2}(q)-H^{2}(-q) & =4 q^{3} \frac{\left(q^{8} ; q^{8}\right)_{\infty}}{\left(q^{2} ; q^{2}\right)_{\infty}^{2}}\left[q^{40}, q^{4}, q^{36} ; q^{40}\right]_{\infty} .
\end{aligned}
$$

Proof. Rewriting

$$
H(q) \pm H(-q)=\frac{\left\langle-q^{3} ; q^{10}\right\rangle_{\infty} \pm\left\langle q^{3} ; q^{10}\right\rangle_{\infty}}{\left\langle q^{2}, q^{7},-q^{7} ; q^{10}\right\rangle_{\infty}}=\frac{\left\langle-q^{3},-q^{13} ; q^{20}\right\rangle_{\infty} \pm\left\langle q^{3}, q^{13} ; q^{20}\right\rangle_{\infty}}{\left\langle q^{2}, q^{7},-q^{7} ; q^{10}\right\rangle_{\infty}}
$$

and then applying Proposition 3, we derive the first two identities stated in the example. The third one follows from the product of the first two. 
EXAMPLE 21 (Robins [20, Eqs. 1.25-1.26] for the first two identities).

$$
\begin{aligned}
G^{2}(q) H\left(q^{2}\right)+G\left(q^{2}\right) H^{2}(q) & =2 \frac{\left(q^{10} ; q^{10}\right)_{\infty}^{2}\left\langle q^{3}, q^{4} ; q^{10}\right\rangle_{\infty}}{(q ; q)_{\infty}\left(q^{2} ; q^{2}\right)_{\infty}\left(q^{5} ; q^{10}\right)_{\infty}} \\
G^{2}(q) H\left(q^{2}\right)-G\left(q^{2}\right) H^{2}(q) & =2 q \frac{\left(q^{10} ; q^{10}\right)_{\infty}^{2}\left\langle q, q^{2} ; q^{10}\right\rangle_{\infty}}{(q ; q)_{\infty}\left(q^{2} ; q^{2}\right)_{\infty}\left(q^{5} ; q^{10}\right)_{\infty}} \\
G^{4}(q) H^{2}\left(q^{2}\right)-G^{2}\left(q^{2}\right) H^{4}(q) & =4 q \frac{\left(-q^{5} ; q^{5}\right)_{\infty}^{3}\left(q^{10} ; q^{10}\right)_{\infty}^{3}}{(q ; q)_{\infty}\left(q^{2} ; q^{2}\right)_{\infty}^{2}}
\end{aligned}
$$

Proof. Rewriting

$$
G^{2}(q) H\left(q^{2}\right) \pm G\left(q^{2}\right) H^{2}(q)=\frac{\left\langle-q, q^{2} ; q^{5}\right\rangle_{\infty} \pm\left\langle q,-q^{2} ; q^{5}\right\rangle_{\infty}}{\left\langle q, q^{2} ; q^{5}\right\rangle_{\infty}\left\langle q^{2}, q^{4} ; q^{10}\right\rangle_{\infty}}
$$

and then applying Proposition 3, we derive the first two identities stated in the example. The third one follows from the product of the first two.

EXAMPLE 22 (Ramanujan's identities).

$$
\begin{aligned}
G(q) H(-q)+G(-q) H(q) & =\frac{2}{\left(q^{2} ; q^{4}\right)_{\infty}^{2}} \\
G(q) H(-q)-G(-q) H(q) & =\frac{2 q}{\left(q^{2} ; q^{2}\right)_{\infty}}\left[q^{10},-q^{10},-q^{10} ; q^{10}\right]_{\infty} \\
G^{2}(q) H^{2}(-q)-G^{2}(-q) H^{2}(q) & =4 q \frac{\left(-q^{2} ; q^{2}\right)_{\infty}^{2}}{\left(q^{2} ; q^{2}\right)_{\infty}}\left[q^{10},-q^{10},-q^{10} ; q^{10}\right]_{\infty} .
\end{aligned}
$$

For the first two identities, see [4, Eqs. 3.24-3.25], [6, Eqs. 23-24] and [24, Eqs. 5-6].

Proof. Rewriting

$$
G(q) H(-q) \pm G(-q) H(q)=\frac{\left\langle-q, q^{7} ; q^{10}\right\rangle_{\infty} \pm\left\langle q,-q^{7} ; q^{10}\right\rangle_{\infty}}{\left\langle q^{2}, q^{6} ; q^{10}\right\rangle_{\infty}\left\langle q^{2}, q^{6} ; q^{20}\right\rangle_{\infty}}
$$

and then applying Proposition 3, we derive the first two identities stated in the example. The third one follows from the product of the first two.

According to Theorem 1, it is easy to check the following difference equations:

$$
\begin{aligned}
& \left\langle-q,-q,-q^{4},-q^{4} ; q^{5}\right\rangle_{\infty}-\left\langle q, q, q^{4}, q^{4} ; q^{5}\right\rangle_{\infty}=8 q\left\langle-q^{2} ; q^{5}\right\rangle_{\infty}\left(-q^{5} ; q^{5}\right)_{\infty}^{6} \\
& \left\langle-q^{2},-q^{2},-q^{3},-q^{3} ; q^{5}\right\rangle_{\infty}-\left\langle q^{2}, q^{2}, q^{3}, q^{3} ; q^{5}\right\rangle_{\infty}=8 q^{2}\left\langle-q ; q^{5}\right\rangle_{\infty}\left(-q^{5} ; q^{5}\right)_{\infty}^{6} .
\end{aligned}
$$

In terms of Ramanujan's partition functions, they may be expressed as two interesting identities.

EXAmPle 23 (Two identities on Ramanujan functions).

$$
\begin{aligned}
G^{8}(q)-G^{4}\left(q^{2}\right) & =8 q\left(-q^{5} ; q^{5}\right)_{\infty}^{6} \frac{H(q)}{H\left(q^{2}\right)} G^{4}(q) G^{4}\left(q^{2}\right) \\
H^{8}(q)-H^{4}\left(q^{2}\right) & =8 q^{2}\left(-q^{5} ; q^{5}\right)_{\infty}^{6} \frac{G(q)}{G\left(q^{2}\right)} H^{4}(q) H^{4}\left(q^{2}\right) .
\end{aligned}
$$


EXAMPLE 24 (Robins [20, Eq. 1.27]).

$$
G^{3}(q) H\left(q^{3}\right)-G\left(q^{3}\right) H^{3}(q)=\frac{3 q\left(q^{15} ; q^{15}\right)_{\infty}^{3}}{(q ; q)_{\infty}\left(q^{3} ; q^{3}\right)_{\infty}\left(q^{5} ; q^{5}\right)_{\infty}}
$$

Proof. With $\omega=\exp (2 \pi i / 3)$, as defined previously, rewrite the difference

$$
G^{3}(q) H\left(q^{3}\right)-G\left(q^{3}\right) H^{3}(q)=\frac{\left\langle q^{2}, q^{2}, q \omega, q \omega^{2} ; q^{5}\right\rangle_{\infty}-\left\langle q, q, q^{2} \omega, q^{2} \omega^{2} ; q^{5}\right\rangle_{\infty}}{\left\langle q, q^{2} ; q^{5}\right\rangle_{\infty}^{2}\left\langle q^{3}, q^{6} ; q^{15}\right\rangle_{\infty}} .
$$

The numerator factorizes into

$$
q\left\langle q, q^{3}, \omega, \omega^{2} ; q^{5}\right\rangle_{\infty}=3 q \frac{(q ; q)_{\infty}\left(q^{15} ; q^{15}\right)_{\infty}^{2}}{\left(q^{5} ; q^{5}\right)_{\infty}^{3}}
$$

thanks to Theorem 1 with $a=q^{3}, b=c=q, d=q^{2} \omega$ and $e=q^{2} \omega^{2}$.

Finally, we come to a much tough identity to test the power of Theorem 1.

EXAMPLE 25 (Ramanujan's identity: cf. [6, Eq. 5] and [4, Thm. 3.5]).

$$
G\left(q^{16}\right) H(q)-q^{3} G(q) H\left(q^{16}\right)=\left(-q^{2} ; q^{4}\right)_{\infty}
$$

Proof. Write explicitly the difference of Ramanujan functions

$$
\begin{aligned}
& G\left(q^{16}\right) H(q)-q^{3} G(q) H\left(q^{16}\right)=\frac{\left(q^{5} ; q^{5}\right)_{\infty}\left(q^{80} ; q^{80}\right)_{\infty}}{(q ; q)_{\infty}\left(q^{16} ; q^{16}\right)_{\infty}} \\
& \times\left\{\left\langle q ; q^{5}\right\rangle_{\infty}\left\langle q^{32} ; q^{80}\right\rangle_{\infty}-q^{3}\left\langle q^{2} ; q^{5}\right\rangle_{\infty}\left\langle q^{16} ; q^{80}\right\rangle_{\infty}\right\}
\end{aligned}
$$

The last difference in the braces $\{\cdots\}$ may be reformulated in terms of factorials in base $q^{20}$ as:

$$
\begin{aligned}
\mathrm{Eq}(3.25 \mathrm{~b})=\langle & \left\langle q, q^{4}, q^{6}, q^{9}, q^{8},-q^{8}, i q^{8},-i q^{8} ; q^{20}\right\rangle_{\infty} \\
& \quad-q^{3}\left\langle q^{2}, q^{3}, q^{7}, q^{8}, q^{4},-q^{4}, i q^{4},-i q^{4} ; q^{20}\right\rangle_{\infty} \\
= & \frac{\left(q^{4} ; q^{4}\right)_{\infty}}{\left(q^{20} ; q^{20}\right)_{\infty}}\left\{\left\langle q, q^{6}, q^{9},-q^{8}, i q^{8},-i q^{8} ; q^{20}\right\rangle_{\infty}\right. \\
& \left.-q^{3}\left\langle q^{2}, q^{3}, q^{7},-q^{4}, i q^{4},-i q^{4} ; q^{20}\right\rangle_{\infty}\right\} .
\end{aligned}
$$

According to four factorizations

$$
\begin{aligned}
\left\langle q^{6} ; q^{20}\right\rangle_{\infty} & =\left\langle q^{3},-q^{3}, q^{7},-q^{7} ; q^{20}\right\rangle_{\infty} \\
\left\langle-q^{8} ; q^{20}\right\rangle_{\infty} & =\left\langle i q^{4},-i q^{4}, i q^{6},-i q^{6} ; q^{20}\right\rangle_{\infty} \\
\left\langle q^{2} ; q^{20}\right\rangle_{\infty} & =\left\langle q,-q, q^{9},-q^{9} ; q^{20}\right\rangle_{\infty} \\
\left\langle-q^{4} ; q^{20}\right\rangle_{\infty} & =\left\langle i q^{2},-i q^{2}, i q^{8},-i q^{8} ; q^{20}\right\rangle_{\infty}
\end{aligned}
$$


we have the following further reduction

$$
\begin{aligned}
\operatorname{Eq}(3.25 b)= & \frac{\left(q^{4} ; q^{4}\right)_{\infty}}{\left(q^{20} ; q^{20}\right)_{\infty}}\left\langle q, q^{3}, q^{7}, q^{9}, i q^{4},-i q^{4}, i q^{8},-i q^{8} ; q^{20}\right\rangle_{\infty} \\
& \times\left\{\left\langle-q^{3},-q^{7}, i q^{6},-i q^{6} ; q^{20}\right\rangle_{\infty}-q^{3}\left\langle-q,-q^{9}, i q^{2},-i q^{2} ; q^{20}\right\rangle_{\infty}\right\} \\
= & \frac{\left(q^{4} ; q^{4}\right)_{\infty}}{\left(q^{20} ; q^{20}\right)_{\infty}} \frac{\left(q ; q^{2}\right)_{\infty}}{\left(q^{5} ; q^{10}\right)_{\infty}} \frac{\left(-q^{8} ; q^{8}\right)_{\infty}}{\left(-q^{40} ; q^{40}\right)_{\infty}} \\
& \times\left\{\left\langle-q^{3},-q^{7}, i q^{14},-i q^{14} ; q^{20}\right\rangle_{\infty}-q^{3}\left\langle-q^{9},-q^{19}, i q^{2},-i q^{2} ; q^{20}\right\rangle_{\infty}\right\} .
\end{aligned}
$$

In Theorem 1, taking $a \rightarrow-q^{19}, b \rightarrow-q^{3}, c \rightarrow-q^{7}, d \rightarrow i q^{14}, e \rightarrow-i q^{14}$ and $q \rightarrow$ $q^{20}$, we can factorize the last line into

$$
\left\langle q^{12}, q^{16}, i q^{5},-i q^{5} ; q^{20}\right\rangle_{\infty}=\frac{\left(-q^{10} ; q^{20}\right)_{\infty}}{\left(q^{20} ; q^{20}\right)_{\infty}}\left(q^{4} ; q^{4}\right)_{\infty}
$$

which leads us to the following equation.

$$
\begin{aligned}
\mathrm{Eq}(3.25 \mathrm{~b}) & =\frac{\left(q^{4} ; q^{4}\right)_{\infty}^{2}}{\left(q^{20} ; q^{20}\right)_{\infty}} \frac{\left(q ; q^{2}\right)_{\infty}}{\left(q^{5} ; q^{10}\right)_{\infty}} \frac{\left(-q^{8} ; q^{8}\right)_{\infty}}{\left(-q^{40} ; q^{40}\right)_{\infty}} \frac{\left(-q^{10} ; q^{20}\right)_{\infty}}{\left(q^{20} ; q^{20}\right)_{\infty}} \\
& =\frac{\left(q^{4} ; q^{4}\right)_{\infty}^{2}\left(q ; q^{2}\right)_{\infty}\left(-q^{8} ; q^{8}\right)_{\infty}}{\left(q^{5} ; q^{5}\right)_{\infty}\left(q^{80} ; q^{80}\right)_{\infty}}
\end{aligned}
$$

Substituting this into (3.25a) and (3.25b) and then simplifying the result, we derive the identity stated in the example.

From this example, we observe that when applying Theorem 1, it is sometimes not a routine matter to identify equivalent products (cf. Bailey [2]) of theta functions.

\section{REFERENCES}

1. G. E. Andrews, The theory of partitions in Encyclopedia of Mathematics and its Applications (Vol. 2) (Addison-Wesley, Reading, MA, 1976).

2. W. N. Bailey, On the simplification of some identities on the Rogers-Ramanujan type, Proc. London Math. Soc. (3) 1 (1951), 217-221.

3. B. C. Berndt, Ramanujan's Notebooks (Part III) (Springer-Verlag, 1989).

4. B. C. Berndt, G. Choi, Y. S. Choi, H. Hahn, B. P. Yeap, A. J. Yee, H. Yesilyurt and J. Yi, Ramanujan's forty identities for the Rogers-Ramanujan functions, Memoirs of American Mathematical Society, to appear.

5. B. C. Berndt, S. H. Chan, Z. G. Liu and H. Yesilyurt, A new identity for $(q ; q)_{\infty}^{10}$ with an application to Ramanujan's partition congruence modulo 11, Quart. J. Math. Oxford Ser. 2 55 (2004), 13-30.

6. B. J. Birch, A look back at Ramanujan's notebooks, Math. Proc. Cambridge Philos. Soc. 78 (1975), 73-79.

7. R. Blecksmith, J. Brillhart and I. Gerst, Parity results for certain partition functions and identities similar to theta function identities, Mathematics of Computation 48 (177) (1987), $29-38$. 1987)

8. J. M. Borwein and P. B. Borwein, $\pi$ and the AGM (John Wiley \& Sons, New York,

9. J. M. Borwein, P. B. Borwein and F. G. Garvan, Some cubic modular identities of Ramanujan, Trans. Amer. Math. Soc. 343(1) (1994), 35-47. 
10. W. Chu, A trigonometric identity with its $q$-analogue, Amer. Math. Monthly 99(5) (1992), Problem 10226; ibid 103:2 (1996), Solution.

11. W. Chu, Theta function identities and Ramanujan's congruences on partition function, Quart. J. Math. Oxford Ser. 2 56(4), (2005), 491-506.

12. J. A. Ewell, Completion of a Gaussian derivation, Proc. Amer. Math. Soc. 84(2) (1982), 311-314.

13. J. A. Ewell, Arithmetical consequences of a sextuple product identity, Rocky Mountain J. Math. 25(4) (1995), 1287-1293. 423.

14. J. A. Ewell, A note on a Jacobian identity, Proc. Amer. Math. Soc. 126(2) (1998), 421-

15. H. M. Farkas and I. Kra, Partitions and theta constant identities, in Analysis, geometry, and Number thoery: The mathematics of Leon Ehrenpreis, Contemp. Math. 251 (2000), 197-203.

16. G. Gasper and M. Rahman, Basic hypergeometric series (second edition) (Cambridge University Press, 2004).

17. B. Gordon, Some identities in combinatorial analysis, Quart. J. Math. Oxford Ser. 2 (12) (1961), 285-290.

18. Z. G. Liu, A three-term theta function identity and its applications, Advances in Math. 195 (2005), 1-23.

19. S. Kongsiriwong and Z. G. Liu, Uniform proofs of $q$-series-product identities, Results in Math. 44 (2003), 312-339.

20. S. Robins, Arithmetic properties of modular forms, $\mathrm{Ph}$. D Thesis, University of California at Los Angeles (1991).

21. I. J. Rogers, Second memoir on the expansion of certain infinite products, Proc. London Math. Soc. 25 (1894), 318-343.

22. L. C. Shen, On the products of three theta functions, Ramanujan Journal 3 (1999), $343-357$.

23. S. Ole. Warnaar, A generalization of the Farkas and Kra partition theorem for modulo 7, J Combinatorial Theory (Series A) 110 (2005), 43-52.

24. G. N. Watson, Proof of certain identities in combinatory analysis, J. Indian Math. Society 20 (1933), 57-69.

25. E. T. Whittaker and G. N. Watson, A course of modern analysis [Fourth Edition] (Cambridge University Press, 1952). 